\title{
25 ANNI DI CRIMINALIZZAZIONE RAZZISTA IN EUROPA
}

\author{
Salvatore Palidda ${ }^{1}$
}

\begin{abstract}
Il testo analizza la criminalizzazione razzista degli immigrati in Europa e negli Stati Uniti come fenomeno intrinseco della globalizzazione neoliberista a livello locale, nazionale e mondiale, in particolare dal 1990 al 2015. Attraverso ricerche degli ultimi 20 anni, I'articolo fa riferimento alla teoria interazionista della costruzione sociale. L'autore mostra come il fenomeno è legato al proibizionismo delle migrazioni e al protezionismo delle nazionalità dominanti e anche alla produzione di inferiorizzazione e neo-schiavitù dei migranti, dei rifugiati e perfino di molti autoctoni. La criminalizzazione razzista degli immigrati ha dunque una "funzione specchio" rivelando la brutale deriva dei dispositivi e delle pratiche di dominio dei dominanti, spesso interiorizzati dai dominati (autoctoni e gli immigrati). Il caso italiano è analizzato come caso rivelatore del governo liberista delle migrazioni contemporanee.
\end{abstract}

Parole chiave: criminalizzazione razziale, la globalizzazione del neoliberismo, inferiorizzazione, neo-schiavitù.

\section{Riflessione sulle ricerche sulla criminalizzazione razzista degli immigrati in Europa}

Nella lunga storia delle immigrazioni si sono sempre riprodotti periodi o congiunture di criminalizzazione razzista e persecuzioni violente degli immigrati, alternati a periodi di quasi pacifico inserimento e di massiccia regolarizzazione di milioni di stranieri nei paesi di arrivo. Basta guardare la storia delle migrazioni italiane interne e internazionali da prima dell'unità d'Italia sino agli anni sessanta, una storia che può essere considerata paradigmatica di tutte le migrazioni di ieri e di oggi ${ }^{2}$. Ma, come vedremo dopo, oggi si rischia di assistere al peggio, cioè a una sorta di tanatopolitica riservata ai migranti in particolare da parte dei paesi europei.

Facoltà di Scienze della Formazione dell'Università degli Studi di Genova. Genova, Italia.

2 Si veda il film documentario Pane Amaro (in italiano: <https://www.youtube.com/watch?v=MK66S1ji_ PY > ; in inglese: <https://www.youtube.com/watch? $v=$ wuEiEXxE5zl) $>$; Parte dell'emigrazione italiana (anche Brasile): <https://www.youtube.com/watch? $v=8 X \mathrm{~d} 3 \mathrm{~J} 0 \mathrm{OyH} \mathrm{xw}>$; Immigranti del Sud Italia al Nord Italia: <https://www.youtube.com/watch?v=RUu9ZEa69KA>; Emigrazione nel secondo 
Quasi sempre il carattere sfavorevole o favorevole all'immigrazione è dipeso dall'andamento economico o da particolari congiunture politiche. Nei periodi di crescita economica e quindi di forte bisogno di manodopera l'immigrazione era incitata e aiutata all'integrazione regolare e ciò sino agli anni ottanta. Le ricerche sulla devianza e la criminalità degli immigrati erano rare e interessavano solo alcuni criminologi clinici e psichiatri ${ }^{3}$ che non mancavano di adottare un approccio più o meno lombrosiano, mentre qualche antropologo democratico invocava il relativismo culturale ${ }^{4}$; dopo, la teoria interazionista della costruzione sociale mostrava come l'approdo dell'immigrato alla devianza o alla criminalità non è diverso da quello dell'autoctono e cioè dipende dal frame o contesto e dalle molteplici interazioni al suo interno ${ }^{5}$.

Con gli anni novanta si sviluppa invece una grande attenzione a questo argomento affrontato seguendo due principali approcci totalmente opposti6:

- La lettura che si può definire neo-positivista e che, di fatto, è organica al proibizionismo delle migrazioni, al protezionismo e alla chiusura a difesa dei privilegi reali o presunti della cittadinanza nazionale o in Europa europea; quindi una lettura che legittima l'idea che i migranti sono più criminali degli autoctoni dei paesi di immigrazione usando le statistiche come prova indiscutibile ${ }^{7}$; non si riconosce che queste non sono altro che la produzione di un discorso dominante sugli oggetti di tale criminalizzazione, un discorso che oscilla dalla discriminazione al razzismo sino alla postura di guerra contro il nemico; questo si traduce in dispositivi (leggi e direttive) e pratiche delle polizie e di tanti cittadini che contribuiscono alla criminalizzazione cui corrisponde l'inferiorizzazione che fa comodo a una buona parte della società.

- La lettura che spiega la devianza e la criminalità innanzitutto come prodotto della criminalizzazione razzista e comunque di una costruzione sociale che riguarda una piccola minoranza dei migranti, processo che può iniziare

dopoguerra: <http:/www.raistoria.rai.it/articoli/emigrazione-nel-dopoguerra/29031/default.aspx>. Vari saggi di Sanfilippo, Rinauro, Colucci, Gallo, e il sito asei.eu. Fra tante importanti pubblicazioni, vedi BEVILACQUA, Piero, DE CLEMENTI, Antonietta, FRANZINA, Emilio (a cura di). Storia dell'emigrazione italiana; SANFILIPPO, Matteo, CORTI, Paola. Storia d'Italia; COLUCCI, Michele, SANFILIPPO, Matteo, Guida allo studio dell'emigrazione italiana; RINAURO, Sandro. Il cammino della speranza. L'emigrazione clandestina degli. Italiani nel secondo dopoguerra; PALIDDA, Salvatore. Mobilità umane.

3 L'idea che il migrante in quanto "spostato" da un "mondo" a un altro fosse sicuramente soggetto a squilibrio mentale è ancora oggi accreditata da alcuni criminologi, psichiatri e psicologi. Si tratta palesemente di "scienze" ancillari all'ordine voluto da un potere conservatore che vede ogni spostamento come una minaccia alla stabilità e al disciplinamento.

4 A volte discutibile perché giustificava comportamenti o atti criminali anche se invocando giustamente le conseguenze di una sbagliata gestione dello choc fra culture.

5 II riferimento è ad alcuni autori della c.d. seconda scuola di Chicago fra i quali Goffman e H.S. Becker

6 Ricordo che sino al 1994 in Italia non era stata realizzata nessuna ricerca sulla devianza o criminalità degli immigrati. Ringrazio la Fondazione Ismu per avermene dato la possibilità sin da allora, dopo ancor meglio sviluppata grazie al progetto europeo MIGRINF e a quelli che successivamente ho potuto realizzare.

7 Vedi in particolare BARBAGLI, Marzio. Immigrazione e criminalità in Italia. 
nel paese di origine, ma che più spesso si innesca e si sviluppa nel paese di immigrazione. Come vedremo dopo, questa lettura insiste anche sulla grande utilità economica e politica di questa criminalizzazione e sulla assoluta necessità di situare il fenomeno nel contesto del trionfo liberista.

Descriverò queste due letture attraverso i commenti o analisi che entrambe fanno di fatti particolarmente significativi. Farò ricorso anche al confronto fra I'immigrazione negli Stati Uniti e in Europa ma anche in altre zone del mondo perché aiuta molto la comprensione dei fatti.

\section{Dal permissivismo tacito al proibizionismo violento}

Già negli anni Ottanta, in tutto il mondo, il trionfo liberista e la sua globalizzazione provocano innanzitutto una nuova politica e nuove pratiche di gestione delle migrazioni. Dopo lo smantellamento dell'assetto industriale, nei paesi dominanti non c'è più domanda di manodopera stabile e regolare per le grandi e medie fabbriche. Il liberismo è "meno stato più mercato", "meno lacci e lacciuoli", più libertà per gli attori economici. Conseguenza immediata è la crescita molto veloce delle economie semi-sommerse o del tutto al nero che impiegano lavoratori autoctoni, immigrati regolari e immigrati irregolari ("clandestini"). Per il datore di lavoro "medio" I'ideale diventa avere questi tre tipi di lavoratori per poter giocare sul ricatto incrociato fra loro: ai primi si dà di più, ai secondi un po' meno e ai terzi ancora meno e a volte neanche quello; la minaccia per i primi è che se non accettano possono stare a casa perché c'è una lunga fila di altri pronti a prendere il loro posto, lo stesso si fa con i secondi e anche con i terzi che in più sono minacciati di essere consegnati alla polizia e quindi a rischio di espulsione. Da qui a innescare situazioni di vera e propria neo-schiavitù ci passa poco (vedi infra). Ovviamente questo "gioco" si può fare solo se il potere politico ha istituito il dispositivo proibizionista. L'immigrazione regolare diventa quasi impossibile; la trafila è un calvario e i costi morali e materiali ingenti (dall'accesso al visto, a quello al permesso, poi al rinnovo del permesso ecc.); si arriva all'apparente paradosso di esigere dagli immigrati una regolarità e dei requisiti che buona parte degli autoctoni non hanno. Nei fatti, il proibizionismo e le regole di legge sempre più rigide, oltre alla discrezionalità sino al libero arbitrio da parte della polizia, delle autorità locali, dei datori di lavoro e anche dei proprietari di alloggi riproducono immancabilmente immigrazione irregolare. Come si scrive in diversi rapporti al Congresso USA già nei primi anni 2000, senza il contributo degli indocumentados non si avrebbe avuto il successo economico che nei fatti è durato sino al 2007 e dopo, come riconoscono anche i centri di ricerca di grandi banche, non si avrebbe avuto lo stesso superamento della crisi. In altre parole, si conferma così che la neo-schiavitù è molto utile alla massimizzazione dei profitti di cui si nutre l'economia liberista. Ricordiamo che gli immigrati irregolari negli USA hanno superato i tredici milioni già ai tempi di Bush e ancora con Obama, nonostante ogni anno centinaia e a volte migliaia sono 
stati uccisi alla frontiera messicana e oltre un milione sono stati espulsi; secondo il Rapporto $\mathrm{IOM}^{8}$ le stime dei migranti morti tra Messico e Stati Uniti sono allarmanti. Ma, a differenza dell'Europa, va riconosciuto che allo stesso tempo gli Usa hanno integrato una quantità di immigrati senza precedenti ${ }^{9}$ : i cittadini statunitensi sono passati da 250 milioni del 1990 a più di 320 milioni nel 2015 ${ }^{10}$. Essi confermano così il paradigma e quindi le pratiche sui quali è stata costruita tutta la loro storia: un paese che s'è nutrito del continuo arrivo di immigrati (ben al di là dei Quota Acts ecc.) come molla fondamentale per l'edificazione della prima potenza mondiale sul piano militare, economico e politico. Ogni nuovo arrivato sa che deve prima subire umiliazioni, angherie, razzismo e che però avrà le opportunità della "riuscita"... spesso sulla pelle di chi arriva subito dopo di lui e a volte persino dei suoi parenti e compaesani, cioè se ha interiorizzato lo "spirito" americano della sopraffazione dell'altro per affermarsi; si tratta dell'immaginario western e wasp di sterminio degli indiani poi trasformato nel pseudo melting pot che è etnicizzazione per la competizione utile alla riproduzione continua della gerarchizzazione economica e sociale, il tutto integrato nel nazionalismo della prima potenza mondiale (sempre pronti a morire per la supremazia USA) ${ }^{11}$. Chi non si adegua deve essere eliminato, come limone spremuto o scarto di produzione ${ }^{12}$ senza neanche la regola three strikes and you're out, cioè sei fuori prima ancora della terza volta che mostri di non andare bene (secondo chi comanda). Le tesi razziste di Huntington (contro arabi e poi anche latinos) s'inscrivono perfettamente nell'articolazione tra inclusione e rigetto, quindi nell'inferiorizzazione dell'immigrato così costretto a guadagnarsi la salvezza mostrando la sua disponibilità a essere schiavizzato ${ }^{13}$.

8 BRIAN, Tara, LACZKO, Frank/IOM. Fatal Journeys Tracking Lives Lost during Migration.

9 II Census Bureau stima che nel 2050 la popolazione degli USA raggiungerà 397 milioni grazie all'immigrazione, ma soltanto 328 milioni senza immigrazione. Secondo il Pew Research Center nel 2050 i non-ispanici bianchi saranno 47\% della popolazione (erano 67\% nel 2005 e 85\% nel 1960). Dopo gli Stati Uniti, la Francia è il paese la cui popolazione attuale è in maggioranza d'origine immigrata, ma forse lo stesso si può dire dell'Argentina dove gli indigeni sono stati eliminati o sopraffatti dagli immigrati italiani e spagnoli.

${ }^{10}$ Vedi statistiche sui siti ufficiali/federali, quelli dell'OCSE, dell'ONU e in quelli dei centre di ricerca fra i quali I'MPI, la Rand Corporation, il Pew ecc.

11 PALIDDA, Salvatore. Emigration, Immigrations, mobilités, un fait politique total.

12 RAHOLA, Federico. Zone definitivamente temporanee. I luoghi dell'umanità in eccesso; BAUMAN, Zigmunt. Vidas Desperdiçadas (Wasted Lives).

${ }^{13} \mathrm{E}^{\prime}$ importante osservare che l'ideologia wasp può adottare allo stesso tempo pratiche violente e altre apparentemente soft, ma è assolutamente contraria a qualsiasi mescolamento con i nonwasp (anche se si tratta di cattolici: fu il caso degli Irlandesi all'inizio massacrati dai wasp che si definivano "nativi" e oggi è il caso dei Latinos); il razzismo contro i neri non è solo wasp (Ku Klux Klan) ma comune anche agli arabi, asiatici e ai Latinos seguendo la gerarchia razzista che legittimò la tratta degli schiavi; lo sciovinismo e il razzismo francesi e di altri paesi europei non hanno il contenuto ideologico di quello wasp; ricordiamo: la limpieza del sangre e il nazismo hanno avuto uno scopo sia economico che politico a cominciare dall'espropriazione dei beni delle genti di 'razza impura' (per primi gli ebrei) e anche d'ideologie "inaccettabili" (Vedi STOLCKE, Verana; COELLO, Alexandre (eds.). Identidades ambivalentes en América Latina. Siglos XVI-XXI). 
Rispetto agli USA i paesi europei d'immigrazione sono molto più meschini, più proibizionisti e meno integrazionisti, come una sorta di monade ossessionata dalla paura di essere sconvolta dall'arrivo di un qualche diabolico estraneo. Così I'Unione europea (UE) produce sempre più morti fra i migranti che tentano di arrivarvi, meno naturalizzati, meno regolarizzati e più precarietà per gli stessi regolari14. In tutta Europa a 27 (505 milioni di residenti) nel 2012 si hanno meno immigrati regolariche negli USA, cioè 21 milioni (Eurostat) e, dal 1990 meno naturalizzati ${ }^{15}$. Fra i residenti le persone nate nei paesi fuori dall'UE a 27 sarebbero circa 33 milioni. Secondo le stime più affidabili, il totale degli irregolari in Europa non oltrepasserebbe cinque milioni. Ben al di là della differenza tra Europa e Stati-Uniti in materia di welfare, osserviamo che gli USA continuano a puntare sull'immigrazione articolando l'integrazione con le pratiche di selezione violenta, il razzismo e il rigetto. La gestione dell'immigrazione è ancora una volta rivelatrice del fatto che c'è sempre coesistenza di paternalismo, umanitarismo e dominazione violenta, di democrazia e autoritarismo; c'è sempre la possibilità d'anamorfosi dello stato di diritto (passaggio da una modalità all'altra e vice-versa, così come nel gioco di uno specchio deformante ${ }^{16}$ ).

Ciò appare ancora più evidente attraverso l'analisi delle pratiche di gestione quotidiana dell'immigrazione da parte della polizia e di diversi attori privati ${ }^{17}$.

$\mathrm{E}^{\prime}$ assai utile ricordare qualche fatto corrente nell'Europa di prima e dopo caduta del muro.

La prima nuova immigrazione straniera non solo in Italia avvenne negli anni settanta. In Sicilia gli armatori di pescherecci scoprirono che era assai vantaggioso reclutare marinai tunisini ("al nero"); poco dopo lo stesso fecero i padroni dei vasti vigneti per la vendemmia e via via per altri lavori stagionali nell'agricoltura. Nelle città ci finirono invece le filippine come serve 24/24h. Sino al 1990 molti paesi europei non richiedevano visto d'ingresso; era quindi normale che nei paesi dell'Europa occidentale arrivasse qualche migliaia di immigrati soprattutto da Marocco, Tunisia, Algeria e poi alcuni anche dall'Africa sub-sahariana, mentre dall'Asia arrivavano solo filippini e dall'America Latina pochi e in gran parte persone di origini europee più o meno lontane.

Immancabilmente la grande maggioranza di questi immigrati finiva per svolgere lavoro nero, ma si trattava ancora di qualche centinaio di migliaio in tutti i paesi Europei e non si erano ancora innescati i meccanismi di razzializzazione e di criminalizzazione.

\footnotetext{
${ }^{14}$ Fortresse Europe stima a più di venti mila i migranti morti alle frontiere d'Europe tra il 1988 e il 2013, di cui più di nove mila scomparsi in mare, cifra che oggi è cresciuta di circa altri 5 mila morti.

15 Secondo il Rapporto del Dipartimento Affari Economici e Sociali dell'ONU (ONU. International Migration Report 2013, p. 21), i primi dieci paesi che tra il 1990 e il 2013 hanno avuto il più alto numero d'immigrati sono gli Stati Uniti, la Russia, la Germania, I'Arabia Saudita, gli Emirati Arabi Uniti, il Regno Unito, la Francia, il Canada, I'Australia e la Spagna.

16 PALIDDA, Salvatore. L'anamorphose de l'Etat-Nation: le cas italien.

17 PALIDDA, Salvatore. Italians Police Forces into neoliberal turn.
} 
Il grande cambiamento (in negativo) comincia dopo la caduta del muro di Berlino (fatto ovviamente più che positivo). Da notare che non c'è stata mai memoria pubblica degli accordi di Helsinki del 1972 coi quali i paesi europei proclamavano insieme agli Stati Uniti il diritto all'emigrazione (palesemente adottato contro I'URSS): nessun paese ha mai votato il diritto all'immigrazione. Dopo il crollo dell'URSS, la prima reazione della maggioranza dei media fu quella di lanciare l'allarme per I'“invasione" che sicuramente si sarebbe verificata. Alcuni media arrivarono a dare i numeri: 50 milioni in arrivo; un pseudo sondaggio avrebbe chiesto per telefono a un grande campione di abitanti dell'Est se pensavano di andare a Ovest (dopo decenni di totalitarismo e divieto di viaggiare è ovvio che tutti desiderino questo). Ovviamente non ci fu alcuna invasione e a tutt'oggi (a 25 anni di distanza facendo tutti i calcoli) siamo ben lungi dai 50 milioni di immigrati provenienti dai paesi ex-URSS.

Il paese che per prima assorbì più immigrati dall'Est fu (e resta ancora) la Germania, non solo persone di lontane origini tedesche, ma anche gli altri, quasi tutti "clandestini" e collocati nel sommerso che infine ha dilagato in tutta I'Unione europea, ma ufficialmente ignorato, nonostante i Rapporti OIM e di altri organismi internazionali e dello stesso Consiglio d'Europa.

$\mathrm{E}^{\prime}$ ora indispensabile capire un aspetto che purtroppo le scienze politiche e sociali ancora oggi trascurano o non riescono a cogliere.

\section{Dalla destrutturazione economica e sociale al nuovo assetto liberista:} l'emergenza delle paure, la distrazione di massa e l'ascesa del razzismo

All'inizio degli anni 1990 la società locale e nazionale dei diversi paesi europei d'immigrazione era sconvolta dalla fine dell'assetto industriale e dal dilagare della precarizzazione, dalle incertezze del lavoro e del reddito, dal non capire in che futuro sperare (fatto assai sconvolgente per una popolazione che in maggioranza si era abituata a relative certezze "dalla culla alla tomba"). Tanti autori hanno scritto sulla società dell'incertezza, del rischio, delle paure o addirittura delle paranoie ${ }^{18}$. Tutte ciò è stato provocato proprio dalla rivoluzione liberista che però, a differenza delle grandi trasformazioni del passato, non produce distruzione per la ricostruzione di una società pacifica, inclusiva e che pensa al futuro (come teorizzava Schumpeter). Il liberismo è massimizzazione del profitto hic et nunc in ogni campo e a tutti i costi. E passa necessariamente per l'impoverimento di una parte della popolazione anche dei paesi cosiddetti ricchi, passa per riforme che limitano o smantellano il welfare ed erodono i diritti acquisiti nei tanti anni di lotte operaie, popolari e studentesche. Le paure e incertezze sempre più dilaganti sono conseguenza del liberismo; allora, sin dalla fine degli anni Ottanta,

\footnotetext{
${ }^{18}$ Su questa letteratura si vedano i saggi in DAL LAGO, Alessandro, PALIDDA, Salvatore (eds.). Conflict, Security and the Reshaping of Society: The Civilisation of War; PALIDDA, Salvatore (ed.). Razzismo democratico. La persecuzione degli stranieri in Europa.
} 
prima negli USA e poi in Europa, lo stratagemma vincente pensato dai think tanks liberisti e adottato dalle autorità economiche e politiche, pubbliche e private è stato quello di puntare tutto sulla "distrazione di massa" che diventa anche nuova occasione di business. L'operazione è semplice: I'opinione pubblica è bombardata da un'informazione che dice: la colpa di tutti i malesseri e problemi economici e sociali è della criminalità prodotta de immigrati, rom e marginali. La distrazione (rispetto alle responsabilità delle scelte liberiste) funziona benissimo non appena si possono mostrare fatti ben precisi e quindi i responsabili di questi fatti, ossia i nemici di turno ${ }^{19}$. Quando non c'erano gli immigrati questi erano i marginali, i tossicodipendenti, o anche i clochards e le prostitute. Nell'escalation della tolleranza zero si manipolano anche i rapporti numerici: anche se i reati non aumentano, gli arresti e le denunce conoscono invece una crescita abnorme (vedi dopo). Come era avvenuto in passato decine e decine di volte agli italiani e altri emigrati-immigrati in tutti i paesi di immigrazione, il nemico di turno lo si trova facilmente fra i nuovi immigrati. Le polizie sono sollecitate a fare più arresti per confermare il paradigma dell'insicurezza dovuta alla criminalità; la soluzione è presto trovata: il "delitto di faciès" (aspetto o carattere somatico o dizione) è da sempre il reato non scritto e però più facilmente perseguibile e si coniuga con il "delitto d'immigrazione" (istituto col proibizionismo che quindi produce la "doppia pena"). Come avvenuto già negli Stati Uniti, le carceri dei paesi europei d'immigrazione si riempiono di marginali, tossicodipendenti, barboni e sempre più di immigrati. E se si guarda bene il rapporto fra i tassi di carcerazione degli immigrati rispetto a quelli degli autoctoni in Europa si scopre che non sono molto lontani da quello registrato negli Stati Uniti fra i neri e i latinos rispetto ai bianchi.

In Europa, i primi a essere designati come i peggiori sono proprio alcuni originari del Maghreb (i cui discendenti in Francia restano comunque da sempre le principali "prede facili" della polizia e sono maggioranza nelle carceri dove però ufficialmente risultano français). Non appena arrivano gli albanesi e poi i rumeni, il primato passa a questi. Analizzando in dettaglio l'evoluzione del fenomeno si può osservare che c'è stata una vera e propria sostituzione del deviante e delinquente o criminale autoctono con lo straniero, fatto in parte vero per quanto riguarda la manovalanza nello spaccio, furti, piccola ricettazione, manovalanza che comunque continua a lavorare per autoctoni ${ }^{20}$. Ma proprio in virtù della logica liberista, la criminalizzazione degli immigrati e dei rom diventa un business per tanti attori sociali e istituzionali e politicanti. Il giornalista fa carriera per gli scoop sull'immigrato criminale; alcuni dirigenti di polizia anche perché aumentano gli arresti effettuati dai loro subordinati e vanno anche in tv a raccontare le loro performances; gli aspiranti

\footnotetext{
${ }^{19}$ Vedi MANERI, Marcello. I media e la guerra alle migrazioni; PALIDDA, Salvatore (ed.). Governance of Security and Ignored Insecurities in Contemporary Europe.

${ }^{20}$ Questo fatto deriva dalle prime ricerche già anticipate in PALIDDA, Salvatore (ed.). Délit d'immigration. La criminalisation des immigrés en Europe.
} 
politicanti, sui quali nessuno avrebbe scommesso, improvvisamente riscuotono successi elettorali sorprendenti a livello locale e persino a livello nazionale (vedi I'ascesa dei partiti razzisti in tutti i paesi europei). L'accanimento mediatico a sostegno della criminalizzazione razzista va ovviamente al di là di qualsivoglia logica razionale: la stragrande maggioranza dei reati gravi sono appannaggio di autoctoni; la stragrande maggioranza degli immigrati è accusata di reati minori o di reati di immigrazione irregolare; il numero di reati rimane lo stesso o aumento di molto poco (e per via di manipolazioni dei dati ${ }^{21}$ ), ma gli arrestati e i denunciati non cessano di aumentare; dal 1990 a oggi gli immigrati regolari e irregolari sono aumentati di almeno 5 volte ma non solo non c'è stato alcun proporzionale aumento dei reati, ma anche una netta diminuzione in particolare dei reati gravi. Eppure, la maggioranza dei media, tanti politicanti che hanno costruito la loro fortuna essenzialmente gridando contro l'immigrazione e i rom, anche alcuni dirigenti delle polizie e persino alcuni magistrati, periodicamente, hanno continuato a rilanciare I'allarme criminalità degli immigrati, come se si trattasse di "barbari assatanati". In effetti, questi rilanci corrispondono al loro bisogno di rimettere in moto il gioco della distrazione di massa in particolare in congiunture nelle quali c'è il rischio di rivolte popolari a causa della crisi economica; allo stesso tempo tale gioco è assai utile anche a tutta l'industria della paura: se i media gridano che aumentano i furti negli alloggi è alta la possibilità che aumenta la richiesta di sistemi di allarme se non di guardie private o metronotte, nonché di videosorveglianza e di polizze assicurative; e spesso sono proprio le compagnie di assicurazione a finanziare $i$ sondaggi allarmistici che, come ammette uno degli esperti spesso "estorcono" risposte che fanno comodo.

L'altra ragione che spiega la continua riproduzione della criminalizzazione razzista è che ha una doppia funzione: a) riaffermare che l'immigrato non merita pari diritti ma che deve restare sempre sul filo del rasoio di una regolarità difficile da rispettare al cento per cento, notte e giorno e per sempre, senza mai sbagliare neanche una volta (a dimenticare di timbrare il biglietto sul bus, a passare col rosso etc.); b) mostrare agli autoctoni che c'è chi è trattato peggio di loro e che quindi sono superiori a qualcun altro perché titolari della cittadinanza di paesi dominanti. Non è, infatti, per nulla casuale che in Italia, molti razzisti-leghisti che la domenica vanno in piazza a gridare contro gli immigrati, il lunedì mattina vanno a raccogliere i "clandestini" per portarli a lavorare nelle loro fabbrichette o campi o cantieri. In altre parole, si tratta di un razzismo liberista nel senso che serve a negare ogni diritto e quindi a poter disporre di manodopera schiavizzabile. Casi di neo-schiavitù a volte mascherati da semi-legalità e accompagnati da episodi di violenze in particolare nei confronti delle donne, sono emersi in diversi paesi europei fra i quali la Germania, la Spagna e ovviamente I'Italia (vedi infra).

${ }^{21}$ Queste manipolazioni sono state scoperte sia per quanto riguarda I'amministrazione Giuliani a New York, sia per Sarkozy in Francia e anche in Italia (PALIDDA, Razzismo democratico..., op. cit.). 


\section{L'escalation della criminalizzazione razzista in Europa sulla scia di quella degli USA}

Come è noto, negli Stati Uniti l'aumento della popolazione carceraria diventa sempre più forte sin dagli anni settanta, nella cui seconda metà era ancora di mezzo milione di persone mentre nel 2014 si arriva a oltre due milioni e 400 mila, soprattutto maschi e con più del 60 per cento neri (afroamericani / american citizens) e latinos cioè ispanici (soprattutto messicani). I bianchi sono solo uno ogni diciassette detenuti, i neri uno ogni tre e i latinos uno ogni sei. Un afroamericano trentenne ogni dieci è oggi in carcere. La criminalizzazione massiccia di neri è dovuta in particolare all'impatto della cosiddetta "guerra alla droga": due terzi dei detenuti per reati di droga sono persone di colore ${ }^{22}$. La discrezionalità insita nei poteri di polizia scivola facilmente verso il libero arbitrio e persino nell'accanimento razzista che vedi caso si sposa con la corruzione e la stesa criminalità nei ranghi delle polizie (come hanno mostrato tante serie televisive statunitensi, vedi Wire). I recenti assassinii di neri da parte di agenti di polizia in diverse città americane mostra che la "condanna razziale" degli afroamericani non si indebolisce affatto nonostante le promesse e speranze suscitate da Obama. Nei fatti, c'è una precisa correlazione fra la continua riproduzione delle guerre permanenti che cominciano con la prima guerra del Golfo del 1990 e si intensificano dopo l'attentato alle due torri nel 2001. Il discorso di legittimazione della guerra permanente è diventato anche un discorso di continuum delle guerre che si traduce nella tolleranza zero e nella guerra contro ogni comportamento non conforme alla "legge, al decoro e alla morale", definiti secondo la logica dei ceti abbienti, del perbenismo razzista e di odio anche nei confronti dei poveri. A questo s'è aggiunto il fatto che tanti soldati che hanno fatto servizio nei diversi teatri di guerra sono poi confluiti nei ranghi delle polizie producendo quindi una sorta di militarizzazione di queste e così il facile scivolamento verso la gestione violenta del disordine sociale o delle anomie anziché verso una gestione pacifica e di mediazione.

La seria storica delle statistiche carcerarie nell'Europa occidentale mostra che l'aumento continuo dei detenuti è cominciato nel 1990 e di fatto non s'è interrotto nonostante negli ultimi anni anche le autorità di tutela dei diritti umani hanno avuto un po' più di eco mediatico a proposito della sfacciata sproporzione fra il calo dei reati e l'aumento degli arresti ${ }^{23}$. Come si vede dai dati della tabella i paesi europei che oggi hanno il più alto tasso di incarcerazione sono la Spagna e il Regno Unito con la differenza però che nel secondo la percentuale degli stranieri è la più bassa di tutti i paesi europei. La spiegazione è duplice: in UK come in Francia e anche in Belgio e nei Paesi Bassi, cioè in tutti i paesi di "vecchia immigrazione" dove è stata abbastanza praticata la naturalizzazione degli stranieri, i figli dei

\footnotetext{
${ }^{22}$ Vedi <http://www.sentencingproject.org/template/page.cfm?id=122>.

${ }^{23}$ PALIDDA, Razzismo democratico..., op. cit.
} 
naturalizzati, che sono fra i più colpiti dalla criminalizzazione, nelle statistiche figurano come nazionali. L'altra spiegazione è che l'UK e in parte anche la Francia sono paesi che non hanno esitato a essere abbastanza violenti nell'impedire l'arrivo di irregolari ed espellere. $\mathrm{E}^{\prime}$ anche evidente che i paesi con la più alta percentuale di stranieri fra i detenuti siano quelli di più recente immigrazione e anche più esposti all'immigrazione irregolare, cioè Spagna, Italia e Grecia. Anche la Germania e l'Austria sono stati i due paesi di prima destinazione degli irregolari (quando ancora i paesi dell'Est loro confinanti non erano integrati nell'UE). I meccanismi, i dispositivi e le pratiche di criminalizzazione razzista sono assai simili in tutti i paesi, ma è evidente che nei paesi scandinavi e anche in Germania sono alquanto più attenuati. Guardiamo ora un po' più in dettaglio il caso italiano.

\section{La situazione nelle carceri di alcuni paesi europei e negli Stati Uniti}

\begin{tabular}{|c|c|c|c|c|c|}
\hline Paesi & $\begin{array}{l}\text { Tasso su } \\
100 \text { mila } \\
\text { abitanti }\end{array}$ & $\begin{array}{c}\% \text { maschi } \\
\text { stranieri su } \\
\text { totale maschi }\end{array}$ & Paesi & $\begin{array}{l}\text { Tasso su } \\
100 \text { mila } \\
\text { abitanti }\end{array}$ & $\begin{array}{c}\% \text { maschi } \\
\text { stranieri su } \\
\text { totale maschi }\end{array}$ \\
\hline Austria & 104,2 & 49,4 & Finlandia & 55,2 & 15,1 \\
\hline Belgio & 118,8 & 43,2 & Norvegia & 69,9 & 32,9 \\
\hline Francia & 118,3 & 18,2 & Svezia & 57,3 & 30,2 \\
\hline Germania & 77,5 & 28,7 & Danimarca & 67,1 & 26,7 \\
\hline Grecia & 115,5 & 61,4 & Regno Unito & 140,3 & 12,9 \\
\hline Italia & 102,9 & 35,3 & & & \\
\hline Olanda & 60.8 & 20,3 & Stati Uniti d'America & 700,0 & * \\
\hline Portogallo & 86,9 & 18,2 & Brasile $* *$ & 289,0 & $* *$ \\
\hline Spagna & 145,7 & 29,8 & & & \\
\hline Spagna Catalogna & 132,5 & 45,5 & & & \\
\hline Svizzera & 86,9 & 74,3 & & & \\
\hline
\end{tabular}

Source: Prison Stock on 01 Jan. 2014 \& 2015 http://wp.unil.ch/space/space-i/prison-stock-2014-2015/ e, per la percentuale degli stranieri: http://wp.unil.ch/space/files/2015/02/SPACE-I-2013-English.pdf; (*) Negli USA la percentuale degli stranieri in carcere è del 5,5\%, ma, il tasso su 100 mila dei bianchi è 680; per i latino 1.775, per i neri 4.350; il tasso dei neri è quindi 6,4 volte superiore a quello dei bianchi e quello dei latino 2,6 volte. $\left({ }^{* *}\right)$ secondo Prisons Studies, il Brasile ha il tasso più alto dell'America del Sud, seguito da Uruguay, Guyana, Gyana francese, Colombia, Cile, Perù, Suriname, Venezuela, Ecuador, Paraguay, Argentina e Bolivia; per quanto riguarda I'America Centrale il tasso di El Salvador, Belize, Panama e Costa Rica è molto più alto di quello del Brasile mentre quelli del Messico, Honduras, Nicaragua e Guatemala sono più bassi (fonte http://www.prisonstudies.org/highestto-lowest/prison_population_rate?field_region_taxonomy_tid=24).

\section{Il caso italiano}

Reati e persone denunciate o arrestate dalle forze di polizia

\begin{tabular}{|c|c|c|c|c|c|c|c|c|c|}
\hline Anno & Omicidi & Furti & Rapine & Droghe & $\begin{array}{c}\text { Reati non } \\
\text { gravi } * *\end{array}$ & TOTALE & $\begin{array}{c}\text { Total } \\
\text { detenuti }\end{array}$ & Stranieri & $\begin{array}{c}\% \\
\text { Stranieri }\end{array}$ \\
\hline 1990 & 1.773 & 1.605 .329 & 36.830 & 30.691 & 801.257 & 2.501 .640 & 26,150 & 4,017 & 15,4 \\
\hline 2000 & 746 & 1.367 .216 & 37.726 & 34.800 & 727.383 & 2.205 .782 & 53.165 & 15.582 & 29,3 \\
\hline 2010 & 526 & 1.325 .013 & 33.754 & 32.761 & 1.170 .000 & 2.621 .019 & 67.961 & 24.954 & 36,7 \\
\hline 2011 & 550 & 1.460 .205 & 40.549 & 34.034 & 403.735 & 2.763 .012 & 66.897 & 24.174 & 36,1 \\
\hline 2013 & 502 & 1.554 .777 & 44.000 & 33.578 & 459.478 & 2.892 .155 & 62.536 & 21.854 & 35 \\
\hline $2014^{*}$ & 400 & $1.600 .000^{*}$ & 38.280 & $30.000^{*}$ & $460.000^{*}$ & 2.742 .000 & 53.623 & 17.462 & 32,6 \\
\hline
\end{tabular}

Source: ISTAT. Indicatori demografici. Stime per l'anno 2014. < http://www.istat.it/it/archivio/126613>; Dipartimento dell'amministrazione penitenziaria (2015) (http://www.giustizia.it/giustizia/it/mg_1_14_1.wp?previsiousPage=mg_1_1 $4 \&$ contentld $=$ SST165666) ${ }^{*}$ stime; ${ }^{* *}$ cambiamento del sistema statistico e della legge penale. N.B.: la variazione dei 
dati è spesso dovuta a cambiamenti nel sistema statistico. In questi 25 anni ci sono state anche diverse innovazioni normative che ovviamente si ripercuotono sulla "produzione" delle polizie, cioè in aumento di denunce e arresti e quindi incarcerazioni poiché c'è stata una notevole crescita della penalizzazione anziché della depenalizzazione in particolare per reati non gravi ma considerati socialmente intollerabili (spaccio, furto, scippo, immigrazione irregolare, ecc.). Ovviamente questo colpisce soprattutto i marginali, gli immigrati e i rom. Invece, c'è stata una vera e propria clamorosa depenalizzazione dei reati dei cosiddetti "colletti bianchi" (non solo in Italia) oltre che le note leggi ad personam che hanno garantito l'impunità non solo a personalità politiche di primo piano, ma anche a alti burocrati e business men, peraltro sempre dotati di avvocati difensori particolarmente valenti, mentre tanti difensori d'ufficio degli immigrati a volte vanno contro coloro che dovrebbero difendere. Per un'analisi più dettagliata sia del caso italiano che dei più importanti paesi europei e degli Stati Uniti, si veda PALIDDA, Razzismo democratico..., op. cit.

La ricerca sulle attività delle polizie nella gestione dell'immigrazione ha una "funzione specchio"24: è rivelatrice della regolazione economica e sociale e anche delle caratteristiche culturali e politiche di ogni paese. II peso di queste attività sulla produttività totale delle forze di polizia è rilevante, ma in parte realizzata con l'accordo tacito o esplicito di alcuni soggetti privati (nell'interesse comune di questi e delle polizie contro gli immigrati ma a volte anche nei tentativi di aiuto degli immigrati per esempio da parte di associazioni umanitarie quando sono effettivamente tali). La gestione dell'immigrazione riguarda innanzitutto la presenza stabile e instabile dell'immigrazione regolare e irregolare: a volte le polizie fanno finta di non vedere gli irregolari e in altri momenti, invece, agiscono con la repressione esemplare; a volte «chiudono un occhio» per le illegalità degli immigrati regolari, ma in altri casi li si priva della regolarità anche quando non vi è alcun atto illegale (ma per chi può farlo, non è difficile "inventarsi" un reato). Queste pratiche di inclusione ed esclusione appaiono a volte legali, altre volte informali e ancora illegali o persino criminali; possono colpire anche autoctoni, ma gli immigranti sperimentano i comportamenti degli agenti più "zelanti», che di fatto sono anche un messaggio agli autoctoni di conferma della superiorità dei cittadini sugli stranieri, aspetto particolarmente importante in un paese in cui si registra una certa "immigrantofobia" che si confonde anche con una "romfobia".

Si potrebbe obiettare che questi dati sono forse enfatizzati da una congiuntura particolarmente negativa per i rom e per gli immigrati (in genere quando se ne parla negativamente si assimilano ai musulmani che sono spesso tout court amalgamati con i terroristi pseudo-islamisti), fenomeno che s'è esasperato dopo il massacro della redazione di Charlie Hebdo in gennaio 2015. Ma le congiunture di questo tipo si sono ripetute più volte in particolare dal 1990 in poi e ancora di più dopo l'attentato alle due torri di NY dell'11/9/200125.

\begin{tabular}{|l|c|c|c|c|c|c|}
\hline Ostilità a & Italia & Francia & Germania & Regno Unito & Spagna & Polonia \\
\hline Rom & $89 \%$ & $60 \%$ & $34 \%$ & $37 \%$ & $35 \%$ & $48 \%$ \\
\hline Musulmani & $61 \%$ & $24 \%$ & $24 \%$ & $19 \%$ & $42 \%$ & $56 \%$ \\
\hline Ebrei & $21 \%$ & $7 \%$ & $9 \%$ & $7 \%$ & $17 \%$ & $28 \%$ \\
\hline
\end{tabular}

Fonte: Pew Research Center 2015. Disponibile su: <http://www.pewglobal.org/2015/06/02/faith-in-europeanproject-reviving/>.

${ }^{24}$ SAYAD, Abdelmalek. La doppia assenza.

${ }^{25}$ Vedi DAL LAGO, PALIDDA, op. cit.; PALIDDA, Razzismo democratico..., op. cit. 
Il caso italiano può sembrare estremo anche a causa dell'alto tasso di economie sommerse che producono violenza e reazioni violente, fra le quali I'approdo di tanti giovani (maschi e donne) alla devianza o alla delinquenza per rifiuto della neo-schiavitù (l'illusione di guadagno facile con lo spaccio o con i furti e la ricettazione o con la prostituzione). E tutto ciò dipende spesso da una certa diffusione di atteggiamenti sfavorevoli se non razzisti nei ranghi delle polizie che a volte sono anche marchiate da tanti casi di corruzione o connivenza con chi schiavizza gli immigrati.

Le pratiche delle polizie dipendono dal discernimento e quindi dalla discrezionalità che può facilmente scivolare verso il libero arbitrio, ma tutti questi aspetti sono condivisi da una buona parte della popolazione e ovviamente innanzitutto da quella parte che conta, che è amica delle polizie per interesse o perché vuole ordine e disciplina (quasi sempre a comodo suo). La maggioranza dei poliziotti fanno parte di cerchie sociali oltre che di quelle professionali ed è in queste che condividono le categorie positive e negative interiorizzate sin dalla socializzazione primaria e poi secondaria. Se nelle polizie c'è più razzismo che fra la popolazione è quasi sempre per effetto di un reclutamento che ha favorito questo per soddisfare una politica migratoria proibizionista che però deve riprodurre immigrazione irregolare schiavizzabile.

La distinzione fra illecito e lecito, fra tollerabile e intollerabile non sta nella traduzione rigorosa delle norme dei codici penale, civile, amministrativo e stradale ecc., sta nella legittimazione apparentemente popolare che la polizia stabilisce sulla base di quanto considera condiviso dal popolo di suo riferimento. In Italia e anche altrove, il recente tentativo di introdurre nella polizia di stato un'apertura al multiculturalismo è di fatto una piccola operazione di facciata di cui nessuno sa nulla ma che utile per dare un contentino al mondo cattolico e laico pro-immigrati ${ }^{26}$. Nei fatti, non c'è stato alcun effettivo investimento nella formazione delle polizie e i casi di angherie, violenze, torture sono numerosi ${ }^{27}$.

In gennaio 2015, in Italia, c'erano 5 milioni e 73 mila immigranti regolari (8.3\% del totale della popolazione ${ }^{28}$ ), circa 400,000 naturalizzati ${ }^{29}$ e circa 300,000 irregolari ${ }^{30}$. La maggioranza di questa popolazione "ne ha visto di tutti i colori" passando quasi sempre da una fase di irregolarità e poi alla regolarità grazie a ben cinque sanatorie e altre regolarizzazioni (a volte "sottobanco"). Le difficoltà di accesso e

\footnotetext{
${ }^{26}$ MINISTERO DELL'INTERNO. II servizio di polizia per una società multiculturale. Un manuale per la Polizia di Stato.

${ }^{27}$ PALIDDA, Mobilità umane, op. cit.

${ }^{28}$ ISTAT. Indicatori demografici.

${ }^{29}$ Secondo EUROSTAT (2011), in Europa più di 15 milioni d'immigrati hanno acquisito la cittadinanza del paese di residenza (in particolare in Francia, UK, Germania, Paesi Bassi, Belgio, ecc. (PALIDDA, Italians Police Forces..., op. cit.).

${ }^{30}$ I paesi con il più alto numero di regolari sono Germania, Spagna, Italia, UK, Francia, Belgio (circa $81 \%$ di tutti gli immigrati nell'EU a 27).
} 
mantenimento della regolarità e il proibizionismo hanno favorito un mercato criminale per procacciarsi i requisiti necessari: un mercato di brokers, magliari, truffatori amici di alcuni poliziotti, avvocati azzeccagarbugli ecc. Tanti agenti delle polizie sono umanitari, ma la maggioranza condivide un'opinione e anche comportamenti razzisti ${ }^{31}$.

Nel 2011 (non diversamente dagli anni precedenti e seguenti) il numero totale degli immigranti denunciati o arrestati era 283.164 , cioè $31.4 \%$ del totale. Analizzando altri dati si può calcolare che il tasso di denunciati fra gli stranieri è 3,4 volte più alto che tra gli Italiani e il tasso degli arrestati 6.8 volte più alto che fra gli Italiani ${ }^{32}$. Possiamo quindi dire che nel trattamento degli immigrati I'Italia non è da meno degli Stati Uniti (dove il tasso dei neri in carcere è 6,4 volte superiore a quello dei bianchi e quello dei latino 2,6 volte). Lo stesso si può dire per la Francia dove però le statistiche camuffano la realtà indicando solo dati generali di francesi senza distinzione di quelli di origini straniera che è risaputo sono la maggioranza nelle carceri.

La spiegazione della sovra-rappresentazione degli stranieri fra i "clienti" delle polizie sta: a) nel carattere discriminatorio se non razzista delle pratiche di buona parte degli agenti di polizia nel perseguire questa categoria di persone (a cominciare del delitto di faciès), vale a dire nell'abuso della discrezionalità insita nei loro poteri; b) nella costruzione sociale del deviante o delinquente o criminale di origine straniera, processo che in genere si compie nel paese di immigrazione, ossia nell'insieme dei dispositivi e meccanismi che spingono una minoranza di immigrati a scivolare in questa deriva.

Questa tesi si basa su 20 anni di ricerche etnografiche e di accurate analisi di statistiche e documenti fra i quali quelli di processi a stranieri e italiani imputati dello stesso reato. Questo lavoro induce a pensare che non ci sono molte differenze fra la criminalizzazione razzista in Italia, in Europa e negli StatiUniti ${ }^{33}$.

I crimini più frequentemente attribuiti agli stranieri sono furto e rapina $(49,9 \%$ di tutti i furti commessi in Italia nel 2011, il 38,8\% delle rapine e 42,5 di tutto spaccio di droga); ma, molti crimini non sono commessi da stranieri e, in alcuni casi le polizie accusano più di una persona per un solo furto o rapina e ancor più spesso quando si tratta di spaccio di droga; molti furti sono soltanto tentavi di furto che a volte sono trasformati persino in rapine. Quando capita il giudice che rispetta rigorosamente le garanzie di legge per tutti indistintamente, gli stranieri sono spesso assolti.

Come detto prima, è ovvio che nei ranghi degli immigrati ci sono devianti, delinquenti e anche criminali. In base alle biografie di tanti di questi conosciuti

31 Vedi LUNARIA 2014. Cronache di ordinario razzismo. Terzo rapporto sul razzismo in Italia; NAGA. Cittadini senza diritti. Rapporto Naga 2014. Stanno tutti bene.

${ }^{32}$ Per ogni calcolo di percentuali e tassi ho preso in considerazione solo la popolazione degli stranieri regolari e irregolari maschi stranieri e italiani di 18-65 ani d'età.

${ }^{33}$ QUASSOLI, Fabio. Immigrazione uguale criminalità: rappresentazioni di senso comune e pratiche degli operatori del diritto; SBRACCIA, Alvise. Migranti tra mobilità sociale e carcere. Storie di vita e processi di criminalizzazione; DE GIORGI, Alessandro. L'esperimento penale americano; PALIDDA, Razzismo democratico..., op. cit. 
in carcere e fuori dal 1994 a oggi, si può dire che è in Italia che la maggioranza è approdata a una di queste categorie sopra citate. Una costatazione assai semplice ma rivelatrice: laddove la manovalanza deviante, delinquente o criminale è composta soprattutto da autoctoni (per esempio l'Italia del Sud dove c'è un tasso di disoccupazione giovanile che supera il $60 \%$ ) non c'è spazio per gli stranieri e la maggioranza degli arrestati sono nazionali; al contrario laddove il mercato delle attività illegali ha innescato la sostituzione degli italiani con gli stranieri questi sono diventati maggioranza della manovalanza. E' il caso dei venditori di strada abusivi, dello spaccio, dei piccoli furti che sono fra i più arrestati nelle città del nord et del centro Italia. Per quanto riguarda l'evoluzione del numero di detenuti italiani e stranieri come abbiamo già analizzato in recenti pubblicazioni, I'aumento è solo in parte proporzionale a quello degli stranieri regolari e irregolari (che passano da meno di un milione nel 1990 a quasi sei milioni nel 201534).

Guardiamo i reati di cui sono imputati i detenuti italiani e stranieri in carcere ${ }^{35}$.

Detenuti per tipologia di reato al 31 Dicembre 2014*

\begin{tabular}{|c|c|c|c|c|c|c|c|c|c|}
\hline Tipologia di reato & Donne & Straniere & $\begin{array}{c}\% \text { stran.re } \\
\text { su tot. } \\
\text { donne }\end{array}$ & Uomini & Stranieri & $\begin{array}{c}\% \text { stran.ri } \\
\text { su tot. } \\
\text { uomini }\end{array}$ & Totale & $\begin{array}{c}\text { Totale } \\
\text { stranieri }\end{array}$ & $\begin{array}{c}\% \\
\text { stran.ri } \\
\text { Totale }\end{array}$ \\
\hline $\begin{array}{l}\text { Associaz.ne mafiosa } \\
\text { (416bis) }\end{array}$ & 147 & 7 & 4,8 & 6.756 & 101 & 1,5 & 6.903 & 108 & 1,6 \\
\hline Legge droga & 789 & 282 & 35,7 & 18.157 & 6.465 & 35,6 & 18.946 & 6.747 & 35,6 \\
\hline Legge armi & 119 & 16 & 13,4 & 10.059 & 891 & 8,9 & 10.178 & 907 & 8,9 \\
\hline Ordine pubblico & 96 & 50 & 52,1 & 3.051 & 874 & 28,6 & 3.147 & 924 & 29,4 \\
\hline Contro il patrimonio & 1.062 & 364 & 34,3 & 29.225 & 7.776 & 26,6 & 30.287 & 8.140 & 26,9 \\
\hline Prostituzione & 103 & 93 & 90,3 & 737 & 561 & 76,1 & 840 & 654 & 77,9 \\
\hline $\begin{array}{l}\text { Contro la pubblica } \\
\text { amministrazione }\end{array}$ & 136 & 42 & 30,9 & 6.858 & 2.472 & 36,0 & 6.994 & 2.514 & 35,9 \\
\hline Incolumità pubblica & 25 & 4 & 16,0 & 1.476 & 161 & 10,9 & 1.501 & 165 & 11,0 \\
\hline Fede pubblica & 175 & 62 & 35,4 & 4.050 & 1.408 & 34,8 & 4.225 & 1.470 & 34,8 \\
\hline Moralità pubblica & 6 & 1 & 16,7 & 164 & 49 & 29,9 & 170 & 50 & 29,4 \\
\hline Contr la famiglia & 75 & 25 & 33,3 & 1.871 & 459 & 24,5 & 1.946 & 484 & 24,9 \\
\hline Contro la persona & 770 & 289 & 37,5 & 21.397 & 6.355 & 29,7 & 22.167 & 6.644 & 30,0 \\
\hline Contro pers.tà Stato & 15 & 1 & 6,7 & 110 & 26 & 23,6 & 125 & 27 & 21,6 \\
\hline $\begin{array}{l}\text { Contro l'amm.ne } \\
\text { giustizia }\end{array}$ & 279 & 85 & 30,5 & 5.867 & 875 & 14,9 & 6.146 & 960 & 15,6 \\
\hline Economia pubbl. & 16 & 2 & 12,5 & 661 & 15 & 2,3 & 677 & 17 & 2,5 \\
\hline
\end{tabular}

${ }^{34}$ L'evoluzione semestrale dal 1991 al 2015 è qui http://www.giustizia.it/giustizia/it/mg_1_14_1. wp; jsessionid =64E5593CA399C0D608F045001047EA3C.ajpAL01 ? previsiousPage= =4\&contentld=SST165666; tutte le statistiche ufficiali (di tipo "amministrativo" ) si trovano qui: http://www.libertaciviliimmigrazione.interno.it/dipim/site/it/documentazione/statistiche/politiche_ immigrazione_asilo/.

35 I più numerosi fra i detenuti stranieri in Italia sono originari della Romania, Marocco, Albania, Tunisia, Nigeria, Egitto, Algeria e Senegal; i primi quattro costituiscono quasi il $60 \%$ del totale e sono da sempre i più numerosi il che conferma l'idea corrente che fra queste nazionalità ci sono più criminali o la c.d. propensione a delinquere. 


\begin{tabular}{|l|c|c|c|c|c|c|c|c|c|}
\hline Contravvenzioni & 73 & 21 & 28,8 & 3.821 & 617 & 16,1 & 3.894 & 638 & 16,4 \\
\hline Legge stranieri (**) & 49 & 45 & 91,8 & 1.256 & 1.162 & 92,5 & 1.305 & 1.207 & 92,5 \\
\hline $\begin{array}{l}\text { Contro il sent.to e } \\
\text { pietà defunti }\end{array}$ & 36 & 9 & 25,0 & 1.082 & 90 & 8,3 & 1.118 & 99 & 8,9 \\
\hline Altri reati & 77 & 6 & 7,8 & 2.873 & 177 & 6,2 & 2.950 & 183 & 6,2 \\
\hline TOTALI & 4.048 & 1.404 & $\mathbf{3 4 , 7}$ & $\mathbf{1 1 9 . 4 7 1}$ & 30.534 & $\mathbf{2 5 , 6}$ & $\mathbf{1 2 3 . 5 1 9}$ & $\mathbf{3 1 . 9 3 9}$ & $\mathbf{2 5 , 9}$ \\
\hline
\end{tabular}

Fonte: Dipartimento dell'amministrazione penitenziaria - Ufficio per lo sviluppo e la gestione del sistema informativo automatizzato - sezione statistica (disponibile su: <http://www.giustizia.it/giustizia/it/mg_1_14_1.wp;jsessionid=D11 016FDD81D1387A8F6F74E0B2147A5.ajpAL03?previsiousPage $=$ mg_1_14\&contentld $=\overline{\text { SST}} 1112345>$. Consultata il 15/6/2015. $\left(^{*}\right)$ La cifra di ogni categoria di reato corrisponde al numero di soggetti coinvolti. Nel caso in cui ad un soggetto siano ascritti reati appartenenti a categorie diverse egli viene conteggiato all'interno di ognuna di esse. $\mathrm{Ne}$ consegue che ogni categoria deve essere considerata a sé stante e non risulta corretto sommare le frequenze.

Da questa tabella della fine del 2014 possiamo osservare che nonostante in diversi casi gli immigrati siano imputati di più reati e quindi conteggiati più volte, sono imputati del 25,9 del totale dei reati di tutti i detenuti, una percentuale più bassa di quella degli stranieri sul totale dei detenuti a quella data $(32,56 \%)$; si hanno quindi 31.939 reati imputati a 17.462 detenuti stranieri, ossia 1,82 reati a testa mentre per gli italiani 2,53, poiché molti italiani beneficiano dei domiciliari o pene alternative alla detenzione in carcere soprattutto se imputati di reati minori e anche di reati gravissimi ma in attesa di giudizio e considerati non connotati da pericolo di fuga (valutazione discrezionale che dipende molto dal "calibro" dell'avvocato difensore). Infatti, la percentuale dei detenuti stranieri in attesa di giudizio è molto più alta di quella che c'è fra gli italiani. Notiamo subito che non è casuale che la percentuale è più alta fra le donne proprio perché la maggioranza delle straniere detenute sono prostitute di strada mentre è noto agli esperti che le italiane si prostituiscono solo in casa o come escort cioè attraverso circuiti non visibili e quindi non perseguite dalle polizie. La violazione della legge sulle droghe, essenzialmente lo spaccio di strada, è quello in cui gli stranieri hanno la percentuale più alta. II fenomeno dello spaccio praticato da giovani stranieri, soprattutto maghrebini, albanesi, nigeriani ma anche di altri paesi africani prima considerati "immuni" (come il Senegal) e probabilmente in futuro anche da ispanici, ha cominciato a svilupparsi alla fine degli anni 1980 quando questi giovani si sono trovati in situazioni di marginalità e sono stati sollecitati a svolgere questa "attività" dai fornitori di droga che non si fidavano più degli italiani perché tutti tossicodipendenti "irrecuperabili" e spesso confidenti di polizia. Negli anni 2000 il fenomeno s'è diffuso fra altri giovani neo-arrivati e già marchiati dalle illusioni di fare soldi velocemente con lo spaccio. Per quanto riguarda il reato contro l'ordine pubblico, non si tratta quasi mai di manifestazioni non autorizzate o disordini di piazza ma spesso di semplici assembramenti o raduni spontanei che gli immigrati si trovano a praticare perché non dispongono di spazi a tale scopo né di alloggi decenti. Quanto al reato contro il patrimonio si tratta quasi sempre di tentativi di furto più che di furti veri e propri e comunque di un reato predatorio in genere di scarso valore. A volte si tratta persino di un sospetto di furto per evidente "delitto di faciès" (somatico), cioè per chiara 
discriminazione tradotta in criminalizzazione razzista. E un semplice tentativo di furto può anche essere classificato come tentativo di rapina anche senza alcuna arma né propria né impropria ma sol perché il guardiano o padrone del negozio dichiarano con qualche testimone compiacente che il ladro lo ha anche aggredito mentre a volte è esattamente il contrario. Vi sono anche casi clamorosi come quello di un homeless incensurato che tenta di rubare un oggetto del varo di 4,60 euro ed è condannato a sei mesi e migliaia di lire di multa per giunta in appello perché gli avvocati del supermercato esigono una sentenza esemplare data la congiuntura di crisi che rischia di far aumentare gli homeless e quindi i piccoli furti. II reato contro l'amministrazione pubblica per gli stranieri riguarda spesso la documentazione falsa o il non rispetto di un ordine del questore (espulsione) o una contravvenzione non pagata, insomma reati che spesso sono legati direttamente alla condizione di irregolarità e quindi dovuti al proibizionismo. A questo s'accompagna la violazione delle leggi sugli stranieri così come quelli contro l'amm.ne della giustizia, la personalità dello stato. Quanto ai reati contro la fede pubblica, contro la moralità pubblica e contro la famiglia siamo chiaramente nel campo di una definizione discrezionale che si innerva immancabilmente con la discriminazione culturale se non col razzismo di chi stabilisce l'imputazione per tali reati.

Facendo un bilancio di 25 anni di criminalizzazione razzista, si può persino dire che non solo non c'è stato aumento della criminalità che si possa minimamente collegare all'aumento degli immigrati regolari e irregolari, ma anche che quest'ultimo permette di affermare anche "matematicamente" che ha fatto diminuire i reati, tranne quelli dei "colletti bianchi".

\section{Vittime ignorate}

L'aspetto più emblematico, in particolare da 25 anni, è l'aumento della vittimizzazione dei marginali, dei rom e degli immigrati, un fenomeno del tutto ignorato proprio perché le scienze politiche e sociali dominate dalla logica mainstream ossia organiche al proibizionismo e al protezionismo, al contrario hanno costruito ricerche per mostrare il contrario e cioè che i carnefici sarebbero rom, stranieri o marginali autoctoni. Infatti, i cosiddetti sondaggi di vittimizzazione considerati l'ultima grande scoperta scientifica della criminologia e della sociologia della devianza e del controllo sociale, sono realizzati via telefono solo su un campione di persone dotate di telefono fisso, secondo gli abituali criteri dei sondaggi di opinione. Questo fatto, in quanto tale, può essere considerato una "mostruosità scientifica" poiché è arcinoto che: 1) la grande maggioranza della popolazione anche autoctona non ha telefono fisso; anche quelli che ce I'avevano I'hanno tolto per la diffusione quasi totale dei telefoni portatili; 2) proprio le persone più suscettibili di essere vittime di violenze (rom, immigrati irregolari, marginali, ma anche autoctoni deboli come le persone costrette a forme di neo-schiavitù nelle economie sommerse e persino gli studenti fuori sede) non 
hanno telefono fisso né sono prese in considerazione come componente dei campioni di questi sondaggi. Si ottengono quindi risultati assai falsanti non solo per come sono concepite le domande e le risposte proposte, ma perché corrispondono a una specie di campione di una popolazione statica, più o meno abbiente che spesso lascia supporre che i carnefici siano gli "altri", cioè i soggetti socialmente stigmatizzati o considerati asociali se non nemici. A questo corrisponde anche la mancanza di tutela da parte delle forze di polizia di queste vittime ignorate che a loro volta neanche immaginano di poter aver diritto a tale tutela. Si pensi alle donne polacche, rumene o anche italiane e di altre nazionalità che lavorano al nero nei campi e sono spesso soggette a molestie e violenze sessuali e che quando si rifiutano o si rivoltano rischiano persino di essere uccise. Nella ricerca di Sciurba e Carnemolla ${ }^{36}$ si mostra come tante donne polacche senza permesso di soggiorno sono alla mercé degli abusi sessuali dei padroni e caporali, oltre a essere supersfruttate e recluse nelle serre. Ben 119 polacche che lavoravano come raccoglitrici di pomodori sono segnalate come scomparse e si teme siano state uccise visto che se ne sono trovate già 15 corpi martoriati, bruciati, torturati ${ }^{37}$. Ion Cazacu fu bruciato vivo dal suo caporale nel 1999 a $20 \mathrm{~km}$ di Milano, perché chiedeva di essere messo in regola e di essere pagato quanto pattuito ${ }^{38}$. Questi e tanti altri fatti terribili non solo per quanto riguarda assassini razzisti, non si spiegano altrimenti che sempre il prodotto di una società in parte razzista a cui corrisponde anche il comportamento di una parte delle autorità e delle polizie che non si preoccupa di offrire alcuna protezione ai più deboli.

\section{Conclusione}

Contrariamente a quanto gridano i media che di fatto aizzano all'odio verso gli immigrati, dal 1990 a oggi in Europa non si è affatto avuta una immigrazione più importante di quella conosciuta dopo la prima e ancora di più la seconda guerra mondiale (tanto più se si considerano le migrazioni interne che spesso erano trattate al pari di come è oggi trattata quella straniera). E non è anche falso affermare che oggi in Europa c'è più delinquenza e criminalità di quanto se ne registrasse nel XX secolo e ancora più falso dire che quella che oggi si registra sia il prodotto dell'immigrazione e non invece il prodotto di un'anomia provocata dalla dinamica liberista che dal 1990 ha aumentato sempre più la distanza fra ricchezza e povertà, le ingiustizie, gli abusi e la neo-schiavitù (una criminalità comunque di gran lunga meno grave degli anni precedenti e inferiore a quella degli Stati Uniti). Ne consegue che se si osserva e si analizzano

\footnotetext{
${ }^{36}$ SCIURBA, Alessandra (ed.). Due volte sfruttate. Le donne rumene nella fascia trasformata del ragusano.

${ }^{37}$ LEOGRANDE, Alessandro. I desaparecidos polacchi nei campi di pomodoro del Tavoliere delle Puglie (28 gennaio).

${ }^{38}$ FO, Dario, CAZACU, Florina (eds.). Un uomo bruciato vivo. Storia di Ion Cazacu.
} 
tutti i fatti, informazioni e dati con onestà intellettuale si può affermare che la riproduzione della criminalizzazione razzista oggi in Europa come negli Stati Uniti persegue la logica della distrazione di massa che serve innanzitutto a legittimare l'inferiorizzazione economica, sociale e giuridica degli immigrati. Si sperimenta così sulla pelle degli immigrati ciò che poi si pratica anche su buona parte degli stessi autoctoni, ossia una pratica di dominio con lo scopo economico e politico di accentuare i profitti e l'asimmetria di potere. Non stupisce quindi che anche in paesi che oggi cercano di imitare l'esempio di quelli dominanti si inneschi una simile criminalizzazione razzista. Lo si può constatare persino in Marocco ${ }^{39}$ e in altri paesi di immigrazione, come in Brasile e altrove. In altre parole, la globalizzazione del liberismo è immancabilmente anche mondializzazione della criminalizzazione razzismo come delle neo-schiavitù.

\section{Bibliografia}

BARBAGLI, Marzio. Immigrazione e criminalità in Italia. Bologna: il Mulino, 1998.

BAUMAN, Zigmunt. Vidas Desperdiçadas. Jorge Zahar Editor, 2004.

BEVILACQUA Piero; DE CLEMENTI Antonietta; FRANZINA Emilio (eds.). Storia dell'emigrazione italiana. Volume II, Roma: Donzelli Editore, 2002.

BRIAN, Tara; LACZKO, Frank/IOM - International Organization for Migration. Fatal Journeys Tracking Lives Lost during Migration. Geneva: IOM, 2014.

COLUCCl, Michele. Lavoro in movimento. L'emigrazione italiana in Europa 1945-57. Roma: Donzelli, 2008.

COLUCCI, Michele; SANFILIPPO, Matteo. Guida allo studio dell'emigrazione italiana. Viterbo: Sette Città, 2010.

DAL LAGO, Alessandro; PALIDDA, Salvatore (eds.). Conflict, Security and the Reshaping of Society: The Civilisation of War. London: Routledge, 2010.

DE GIORGI, Alessandro. L'esperimento penale americano. In PALIDDA, Salvatore. Razzismo democratico. La persecuzione degli stranieri in Europa. Milano: Agenzia X ed., 2009, p. 36-43.

EDOGUÉ NTANG, Jean Louis; PERALDI, Michel. Un Ancoraggio Discreto. L'insediamento delle Migrazioni Subsahariane nella Capitale Marocchina. In PALIDDA, Salvatore (ed.). II discorso ambiguo sulle migrazioni. Messina: Mesogea, 2011, p. 131-158.

FO, Dario; CAZACU, Florina (eds.). Un uomo bruciato vivo. Storia di Ion Cazacu. Milano: Chiarelettere, 2015.

ISTAT. Indicatori demografici. Stime per l'anno 2014. Istat, 2015.

LEOGRANDE, Alessandro. I desaparecidos polacchi nei campi di pomodoro del Tavoliere delle Puglie (28 gennaio). 2009. Disponibile su: <http://www.unita. it/italia/i-desaparecidos-polacchi-nei-campi-di-pomodoro-del-tavoliere-dellepuglie-1.26660>. Consultato: 08/06/2015.

${ }^{39}$ EDOGUÉ NTANG, Jean Louis, PERALDI, Michel. Un Ancoraggio Discreto. L'insediamento delle Migrazioni Subsahariane nella Capitale Marocchina. 
LEOGRANDE, Alessandro. Uomini e caporali. Viaggio tra i nuovi schiavi nelle campagne del sud. Milano: Mondadori, 2008.

LUNARIA 2014. Cronache di ordinario razzismo. Terzo rapporto sul razzismo in Italia. Disponibile su: <https://www.lunaria.org/wp-content/uploads/2014/10/ impaginato-low.pdf>.

MANERI, Marcello. I media e la guerra alle migrazioni. In PALIDDA, Salvatore. Razzismo democratico. La persecuzione degli stranieri in Europa. Milano: Agenzia X ed., 2009, p. 66-88.

MINISTERO DELL'INTERNO. II servizio di polizia per una società multiculturale. Un manuale per la Polizia di Stato. Ministero dell'Interno, 2004.

MORICE, Alain; POTOT, Swaine (dir.). De l'ouvrier immigré au travailleur sans papier. Les étrangers dans la modernisation du salariat. Parigi: Karthala, 2010.

MURATORE, Maria Giuseppina; TAGLIACOZZO, Giovanna; FEDERICI, Alessandra (eds.). La sicurezza dei cittadini. Reati, vittime, percezione della sicurezza e sistemi di protezione. Istat Informazioni, 18. Roma: Istat, 2004.

NAGA. Cittadini senza diritti. Rapporto Naga 2014. Stanno tutti bene. Disponibile su: <http://www.naga.it/tl_files/naga/comunicati/INVITI/Report_ CitttadiniSenzaDiritti_Rapporto_2014.pdf >.

ONU, Dept. Economic and Social Affairs. International Migration Report 2013. New York: UN, 2013. Disponibile su: <http://www.un.org/en/development/desa/population/ publications/pdf/migration/migrationreport2013/Full_Document_final.pdf $>$.

PALIDDA, Salvatore (ed.). Délit d'immigration. La criminalisation des immigrés en Europe. Bruxelles: COST, 1996.

PALIDDA, Salvatore (ed.). Governance of Security and Ignored Insecurities in Contemporary Europe. London: Ashgate, 2016.

PALIDDA, Salvatore (ed.). Razzismo democratico. La persecuzione degli stranieri in Europa. Milano: Agenzia X, 2009.

PALIDDA, Salvatore. Emigration, Immigrations, mobilités, un fait politique total. Actes Colloque ACSALF \& ASF, Ottawa, in progress.

PALIDDA, Salvatore. Italians Police Forces into neoliberal turn. European Journal of Policing Studies. Settembre, 2015.

PALIDDA, Salvatore. L'anamorphose de I'Etat-Nation: le cas italien. Cahiers Internationaux de Sociologie, v. XCIII, 1992, p. 269-298.

PALIDDA, Salvatore. Mobilità umane. Milano: Cortina, 2008.

PALIDDA, Salvatore. Re-hybridizing the legal and the criminal in all activities at the local, national and global level: a 'political total fact' in the 21st Century neoliberal frame. In SAITTA, Pietro; SHAPLAND, Joanna; VERHAGE, Antoinetta (eds.). Formal, Informal and Criminal Economy: An Outlook on Northern and SouthernEurope. The Hague: Eleven International Publishing, 2013, p. 29-50.

PALIDDA, Salvatore; SANFILIPPO, Matteo (eds.). Emigrazione e organizzazioni criminali. Viterbo: Settecittà, 2012.

QUASSOLI, Fabio. Immigrazione uguale criminalità: rappresentazioni di senso 
comune e pratiche degli operatori del diritto. Rassegna Italiana di Sociologia, v. XL, n. 1, 1999, p. 43-76.

RAHOLA, Federico. Zone definitivamente temporanee. I luoghi dell'umanità in eccesso. Verona: Ombre Corte, 2003.

RINAURO, Sandro. Il cammino della speranza. L'emigrazione clandestina degli. Italiani nel secondo dopoguerra. Torino: Einaudi, 2009.

SANFILIPPO, Matteo; CORTI, Paola. Storia d'Italia. Migrazioni. Annali Einaudi 24. Torino: Einaudi, 2009.

SAYAD, Abdelmalek. La doppia assenza. Milano: Cortina, 2002.

SBRACCIA, Alvise. Migranti tra mobilità sociale e carcere. Storie di vita e processi di criminalizzazione. Milano: Franco Angeli, 2007.

SCIURBA, Alessandra (ed.). Due volte sfruttate. Le donne rumene nella fascia trasformata del ragusano. 2013. Disponibile su: < http://www.meltingpot.org/Duevolte-sfruttate-Le-donne-rumene-nella-fascia.html\#.VYK4JFXtmko >.

STOLCKE, Verana; COELLO, Alexandre (eds.). Identidades ambivalentes en América Latina. Siglos XVI-XXI. Barcelona: Bellaterra, 2008.

\section{Abstract}

\section{5 years of racial criminalization in Europe}

The text analyze the racial criminalization of immigrants in Europe and in the United States as phenomenon intrinsic to the frame of neo-liberalism globalization at local, national and worldwide level, in particular from 1990 to 2015. Through researches of the last 20 years, the article refers on the theory of interactionist social construction. The author show how the phenomenon is linked to prohibitionism of migrations and protectionism of dominant nationalities and also to produce inferiorization and neoslavery of migrants, refugees and also of many autochthonous. The racist criminalization of immigrants has therefore a "mirror function" revealing the brutal drift of devices and practices of dominion often internalized by the dominants as well as by the dominated (autochthonous and migrants). The Italian case study is analysed as emblematic.

Keywords: racial criminalization, globalisation of neo-liberalism, inferiorization, neo-slavery.

Articolo ricevuto il 18/06/2015

Accettato per la pubblicazione il 15/09/2015

Received for publication in June $18^{\text {th }}, 2015$ Accepted for publication in September 15

ISSN impresso 1980-8585

ISSN eletrônico 2237-9843

http://dx.doi.org/10.1590/1980-85852503880004505 